\title{
The Feasibility of Treatment for Skin Diseases Using the Ultrasonic Surgical Aspirator
}

\author{
Chihoko Nakahara $^{1}$, Shiro Niiyama ${ }^{1}$, Satoshi Kajiura ${ }^{1}$, Yoshinori Ito ${ }^{2}$, Noriyoshi Sumiya ${ }^{2}$, \\ Kensei Katsuoka ${ }^{1}$
}

${ }^{1}$ Department of Dermatology, Kitasato University School of Medicine, Sagamihara, Japan; ${ }^{2}$ Department of Plastic and Reconstructive Surgery, Fujigaoka Hospital, Showa University School of Medicine, Yokohama, Japan.

Email: *sniiyama@aol.com

Received December $8^{\text {th }}$, 2012; revised January $10^{\text {th }}, 2013$; accepted January $18^{\text {th }}, 2013$

Copyright (C) 2013 Chihoko Nakahara et al. This is an open access article distributed under the Creative Commons Attribution License, which permits unrestricted use, distribution, and reproduction in any medium, provided the original work is properly cited.

\begin{abstract}
Ultrasound energy liquefies high fluid content tissues, such as epidermis and sweat glands via cavitation, but minimally affects dermis, blood vessels, and nerves at the same energy level. We have used ultrasonic surgical aspirator to treat several skin diseases such as Bowen's disease, wart, prurigo nodularis, and osmidrosis. The ultrasonic surgical aspirator offers an effective and safe method to treat them with few complications. We expect several other skin diseases could likely be cured using ultrasonic surgical aspirator by accumulating additional cases.
\end{abstract}

Keywords: Bowen’s Disease; Osmidrosis; Prurigo Nodularis; Wart

\section{Introduction}

The ultrasonic surgical aspirator (USA) has long been used in the fields of renal surgery [1], neurosurgery [2], and cardiac surgery [3], and is widely available in hospital settings. The ultrasonic energy caused cavitation in high fluid content tissues, such as epidermis and sweat glands, resulting in liquefaction and subsequent removal by suction. However, at the same energy level, solid adjacent structures such as dermis, blood vessels, and nerves were minimally affected [4].

We treat to some skin diseases with this novel and original method using USA, and the results are sufficient. Here, we describe the feasibility of treating skin diseases using USA.

\section{Methods and Case Reports}

The USA used for this treatment is the Sonopet UST2001 (Miwatec Co., Ltd., Kawasaki, Japan). The aspirator is composed of a main unit and a hand piece. The tip of the hand piece consists of titanium, and it has a $2 \mathrm{~mm}$ diameter, $25 \mathrm{kHz}$ frequency, and $300 \mu \mathrm{m}$ maximum oscillation. The operator can adjust the level of oscillation between 0 and 100 . The optimal setting will vary depending on the thickness and hardness of the affected

"Corresponding author. area. Specifically, the thicker and harder the area, the higher is the setting, and we used settings that fell within a range of 40 to 80 . The area around the tip is irrigated with physiological saline, which is suctioned away through the tip itself.

\subsection{Patient 1}

A 72-year-old female had a $50 \times 30 \mathrm{~mm}$, slightly elevated, light red nodule on the neck that had been slowly growing since its appearance 3 years previously (Figure 1). Beforehand, we examined skin biopsy and it was confirmed that Bowen's disease, immature keratinization and irregular acanthosis of the epidermis with clumping cells. A safety margin was marked approximately $5 \mathrm{~mm}$ from the lesion border. A local infiltration anesthesia was administered, while waiting for the effect of local anesthesia, the ultrasonic aspirator was set up. The ultrasonic power was set at $70 \%$, the rate of saline irrigation was set at $40 \mathrm{ml} / \mathrm{min}$, and the suction was $50 \%$. While holding the handpiece like a pen, the operator lightly pressed the tip onto the epidermal surface of the region and moved it around in continuous circular, brush-like motions. At the same time, we cooled the treated area with ice to prevent the burn. After confirmation on visual inspection that the lesion had been totally excised, a portion of the wound was biopsied to confirm the visual findings. Histological 
examination of specimens showed elimination of the epidermis (Figure 2). A steroid and antibiotic ointment was applied to the wound. The course of recovery was uneventful, and the wound was totally epithelialized 2 weeks after the procedure. At 14 months after treatment, no signs of recurrence were observed (Figure 3).

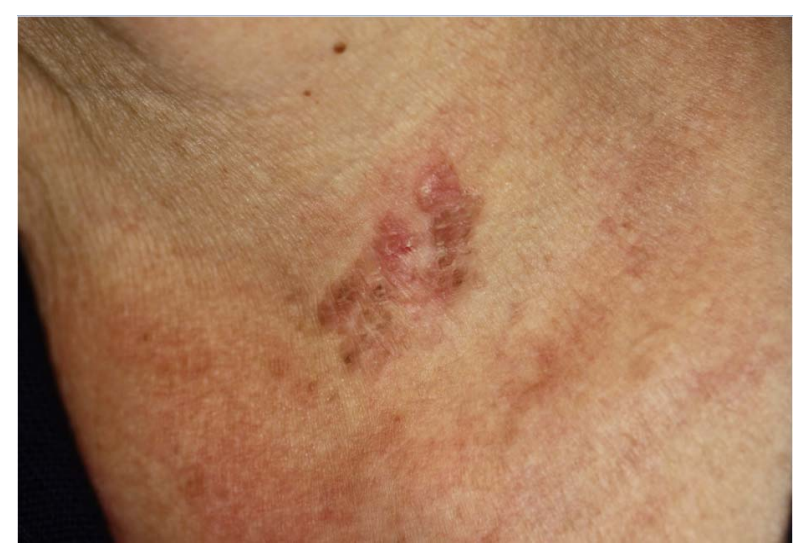

Figure 1. A $50 \times 30 \mathrm{~mm}$ nodule on the neck.

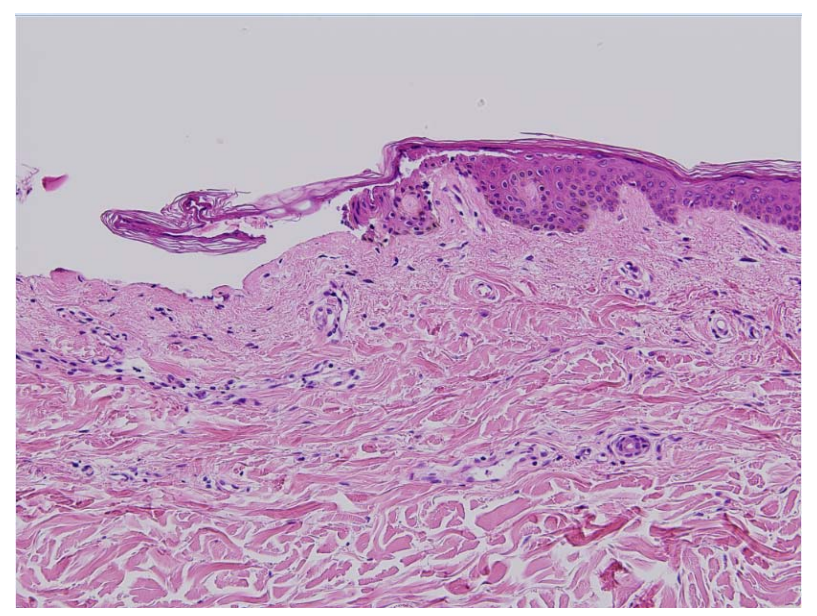

Figure 2. Histological examination showed elimination of the epidermis.

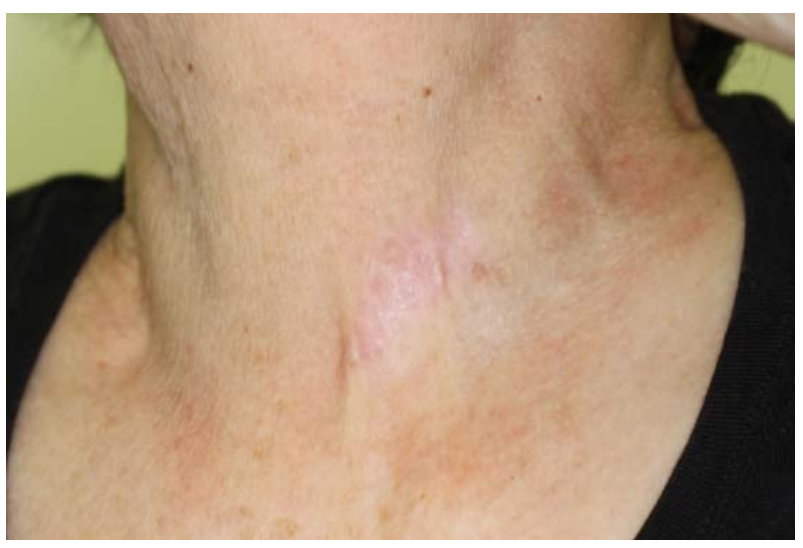

Figure 3. At 14 months after treatment, a slight scar was present but no signs of recurrence.

\subsection{Patient 2}

A 27-year-old male presented with a 5-year history of recalcitrant many warts on the sole (Figure 4). Previously the patient had received treatment with cryotherapy and 5\% imiquimod, however, the treatments were not effective. A local infiltration anesthesia was administered. The ultrasonic power was set at $60 \%$, the rate of saline irrigation was set at $25 \mathrm{ml} / \mathrm{min}$, and the suction was $50 \%$. The thick surface of warts is scraped with a scalpel until a slight amount of blood oozes out. The operator lightly pressed the tip onto the warts and moved it around in continuous circular. Because warts more often recur at the margin than in the center, it is better to scrape slightly more widely around warts. Since the epidermis can detach from the dermis when sites that are allowed to come into contact become white and swell up with fluid, it is grasped and peeled back with forceps, and the cut away with scissors. The remaining epidermis is carefully cut away with the USA. The center often becomes hard, like a clavus, and that portion is always completely scraped away. After arresting the bleeding with an electric coagulator, steroid and antibiotic ointment is applied, and the wound is covered with gauze. Epithelializaition usually occurred in 1 to 2 months and there was no evidence of recurrence on follow-up at 7 months (Figure 5). Histological examination of specimens showed elimination of the epidermis (Figure 6).

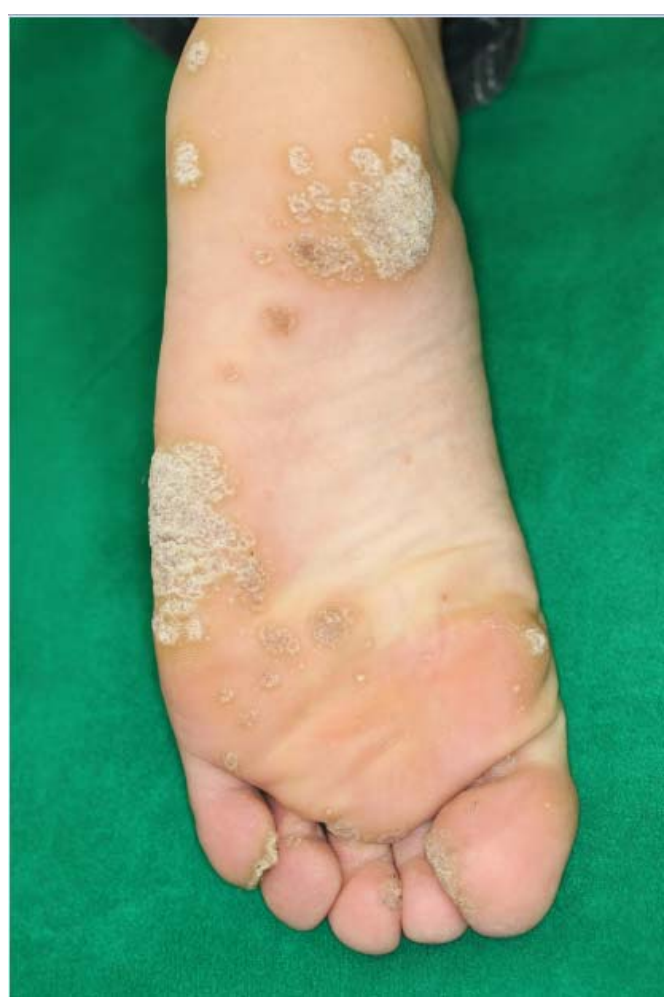

Figure 4. Preoperative appearance of the many warts located on the sole. 


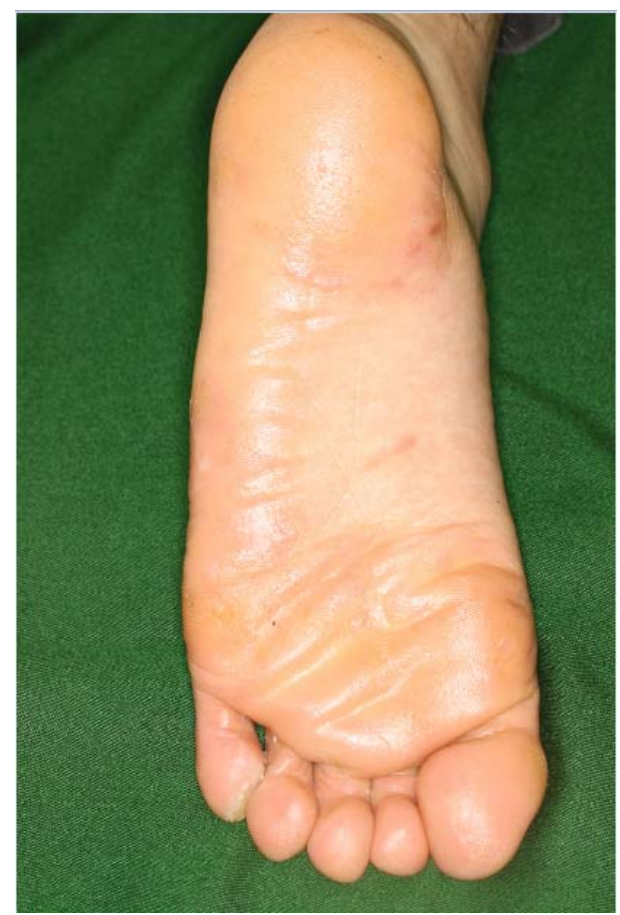

Figure 5. Appearance 7 months after procedure. No scarring or recurrence was present.

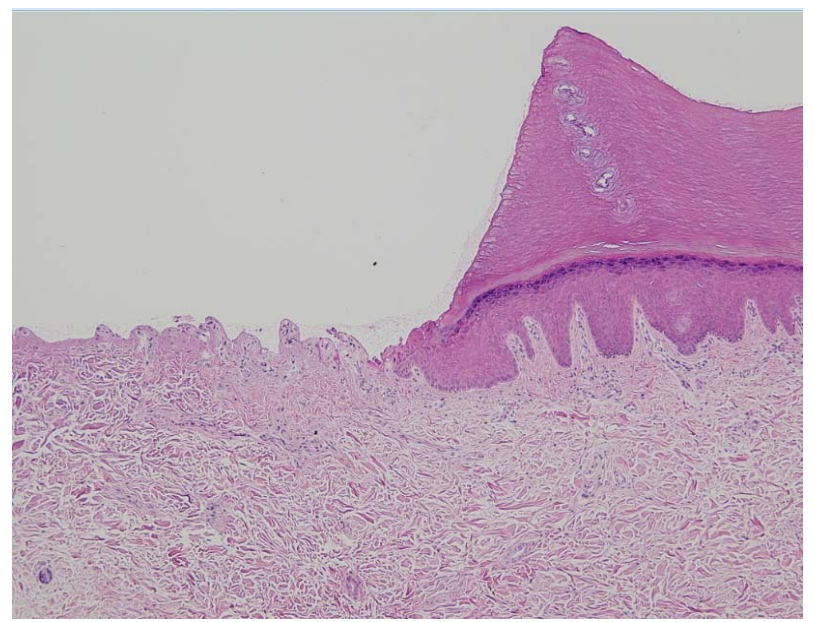

Figure 6. Histological examination of specimens showed elimination of the epidermis.

\subsection{Patient 3}

A 59-year-old female with prurigo nodularis, had intense pruritus for one and a half years. She had multiple nodules, several millimeters in diameter, on her whole body almost. Previously she had received various treatments with the steroid ointment, topical vitamin $\mathrm{D}_{3}$, narrowband UVB, systemic steroid and cyclosporine (3 mg/kg). However, those treatments were not effective. Consequently, we removed many of the nodules on her right elbow (Figure 7) as possible using USA. After administration of local anesthesia, the ultrasonic power was set

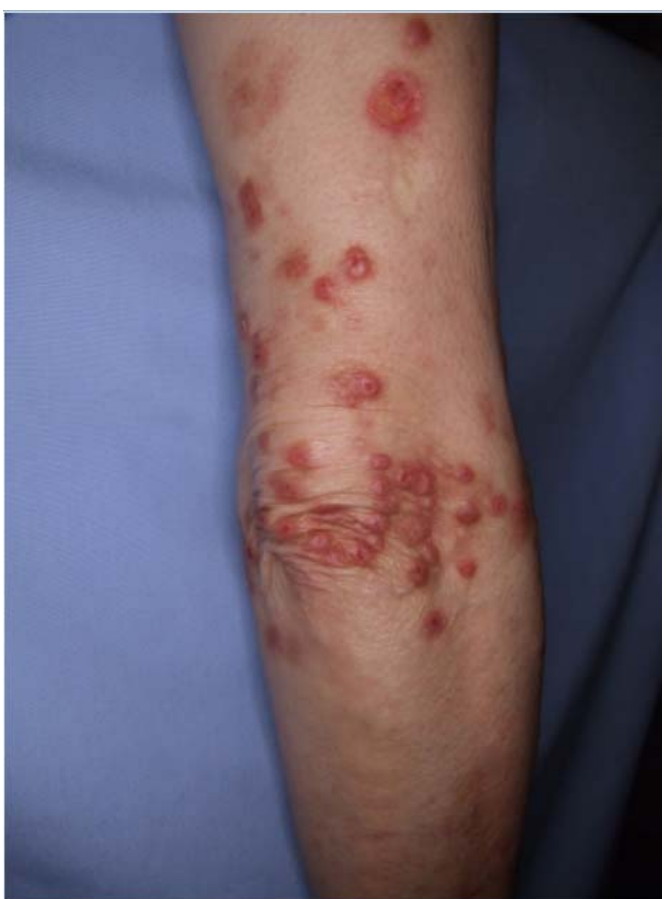

Figure 7. Legions of prurigo nodularis on right elbow.

at $60 \%$, the rate of saline irrigation was set at $20 \mathrm{ml} / \mathrm{min}$, and the suction was $50 \%$. One day after the operation, the itchy sensation was remarkably alleviated. The wound was totally epithelialized 2 weeks after the procedure. Unfortunately, the itchiness on the legion gradually returned about 4 weeks after the procedure.

\subsection{Patient 4}

A 23-year-old female with osmidrosis axillae had received treatments with various external preparations for approximately 3 years. However, all these treatments were ineffective. This patient was placed in a supine position with her arms abducted from the trunk to approximately $100^{\circ}$ to expose the axilla and to avoid injury to the brachial plexus. Markings were made around the location of the axillary hair-bearing area, where the apocrine glands are thought to be located. A local infiltration anesthesia was administered. The ultrasonic power was set at $60 \%$, the rate of saline irrigation was set at 30 $\mathrm{ml} / \mathrm{min}$, and the suction was $50 \%$. A $2 \mathrm{~cm}$ incision was made in the parallel direction of the axillary crease on the central portion of the hair-bearing axillary area (Figure 8). To maintain neurovascular bundles, undermining was performed parallel to the subdermal plexus with scissors. The operator lightly pressed the tip onto the undersurface of the axillary skin and moved it around in continuous circular. Effective removal of apocrine glands was demonstrated by the appearance of hair follicles and dermis. Histological examination of specimens showed elimination of the apocrine glands (Figures 9 and 10). Wounds 


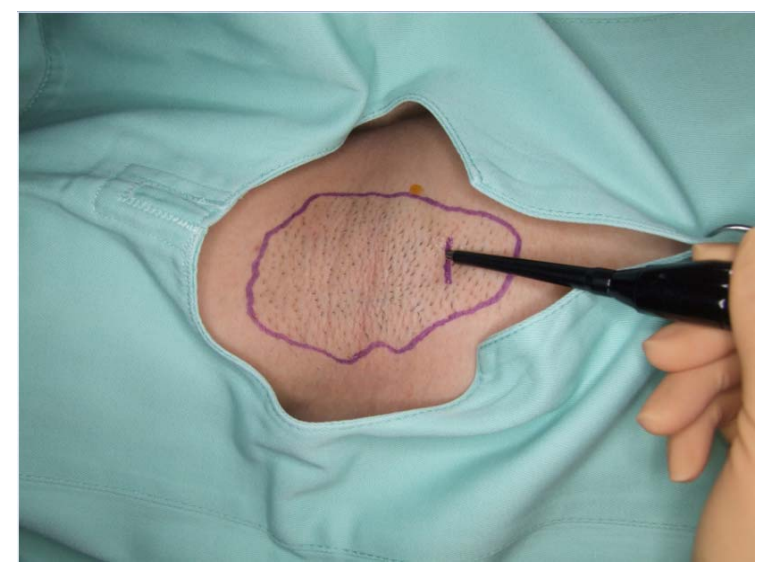

Figure 8. Insert the handpiece through the incision.

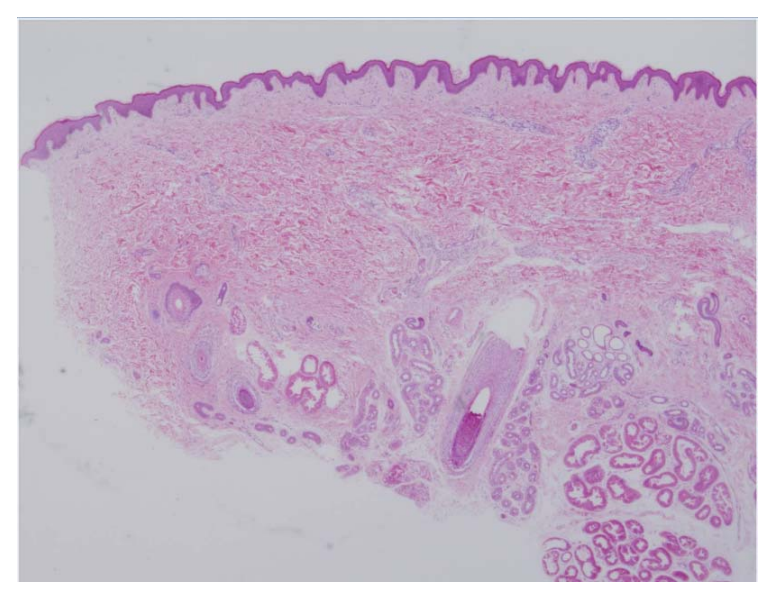

Figure 9. Before ultrasonic aspiration. Normal axillary skin architecture showing apocrine glands.

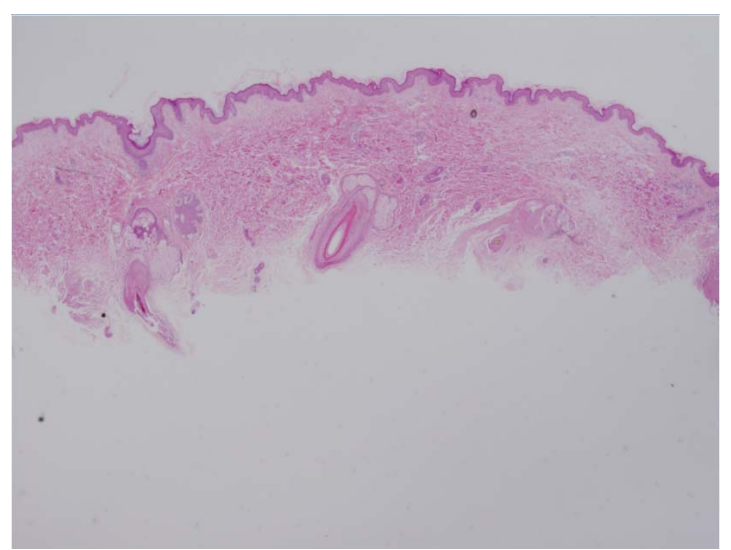

Figure 10. After ultrasonic aspiration. The apocrine glands have been removed.

were closed with sutures followed by coverage with compressive bandages for 3 days. Sutures were removed usually approximately 10 days after surgery, and active shoulder movements were prohibited for 2 weeks postoperatively. Two weeks postoperatively the patient's osmidrosis axillae were evaluated as no malodorousness.

\section{Discussion}

The ultrasonic energy caused cavitation in high fluid content tissues such as epidermis and sweat glands. The cavitation is followed by blasting and liquefaction. As such, ultrasound energy is more effective within epidermis and sweat glands, since these tissues are less dense as compared to more cohesive tissues such as dermis, blood vessels, and nerves. Therefore, it offers the advantages of fairly effortless removal of epidermis and sweat glands, with less bleeding due to minimal destruction of more solid tissue such as blood vessels [4].

Lesions from Bowen's disease are often small and usually can be easily treated by surgical excision and direct closure. However, for patients with lesions that are large and/or located in joint areas, or in surgically problematic areas such as the ears, nose, eyelids, and mucous membranes, surgical excision can become complicated with skin grafting, immobilization of a joint, the use of a general anesthesia, and/or hospitalization sometimes required. All of these factors should be avoided, if at all possible, particularly because most patients with Bowen's disease are elderly, and any one of these circumstances can trigger a decline in general health and well being. Although other nonsurgical options are available, the most widespread being cryotherapy and 5-fluorouracil ointment, the USA offers an option that is effective and inexpensive with few complications.

Because there is currently no cure for human papillomaviruses (HPV) infection, traditional treatment modalities have focused on the destruction of infected tissue through a variety of techniques. These include salicylic acid, podophyllin, bleomycin, cryotherapy, laser therapy, and surgery [5]. Recently, immunomodulatory compounds with antiviral properties have been investigated in HPV therapy. However, such as salicylic acid may cause local irritation and desquamation; bleomycin and imiquimod may offer several side-effects; cryotherapy and laser therapy are painful and may lead to scarring, whereas surgical removal of warts causes localized reactions such as pain, scarring, bleeding, and secondary infections can occur [5]. Moreover, a skin graft or flapformation surgery may be needed, depending on the size and location of the warts. However, the USA offers an option that is very simple, safe, and effective method.

Prurigo nodularis is a benign chronic dermatosis that is often difficult to treat successfully. Topical or intralesional glucocorticoids are the treatment of choice. Other topical treatments such as topical vitamin $\mathrm{D}_{3}$, and topical capsaicin have also been reported. Oral treatments such as cyclosporine and thalidomide have been shown to improve frequently. However, many therapeutic modalities have been used but frequent relapses are common. In our case, one day after using USA, the severe itchiness improved considerably, even the nodules had not com- 
pletely disappeared. This clinical effect of USA on prurigo nodularis is similar to that of cryotherapy. The continued absence of pruritus after cryotherapy may be due to sensory nerve damage and impairment of nerve regeneration. Other effects of cryosurgery on the dermis include marked edema, distortion of cells, and a decrease in capillary circulation with resultant extravasation of erythrocytes [6]. We expect that USA may be effective via the same mechanism as that of cryotherapy.

The cause of osmidrosis has been the topic of several papers, it is generally agreed that the odour originates from bacterial decomposition of sweat secreted from apocrine glands. To treat severe cases, various types of surgical methods have been introduced [7]. Of these procedures, manual shaving had the lowest recurrence rate for malodor. $\mathrm{CO}_{2}$ laser vaporization had results similar to manual shaving except for a clinically higher recurrence rate. Liposuction had advantages such as small invisible scars and the least number of surgical complications, but it had a high rate of dissatisfaction in postoperative malodor. Therefore, removal of subcutaneous apocine glands by manual subdermal shaving is the effective method. However, many problems with wound healing were reported: hematoma, seroma, skin necrosis, and severe scarring. The use of ultrasonic aspirator allowed us to remove apocrine glands with scar less, and the USA offers a safe and effective method.

Even though the USA offers a safer way to treat of skin diseases, it still should be used with care to avoid potential complications such as skin necrosis via a thermal effect. Burns can occur if the operator presses too hard or the device is allowed to remain stationary. However, this problem can be avoided simply by moving the hand piece tip across the region with small, uninterrupted, brush-like strokes. In our department, the treated area is cooled by ice or irrigation with saline maintained at $4^{\circ} \mathrm{C}$ throughout the operation. Topical steroids are applied to the affected area for a few days postoperatively to pre- vent burn.

\section{Acknowledgements}

The authors gratefully thank Dr. Shinsaku Aiba and Mr. Satoshi Kohira for his critical cooperation in preparing this article.

\section{REFERENCES}

[1] R. T. Chopp, B. B. Shah and J. C. Addonizio, "Use of Ultrasonic Surgical Aspirator in Renal Surgery,” Urology, Vol. 22, No. 2, 1983, pp. 157-159. doi:10.1016/0090-4295(83)90499-5

[2] E. S. Flamm, J. Ransohoff, D. Wuchinich and A. Broadwin, "Preliminary Experience with Ultrasonic Aspiration in Neurosurgery," Neurosurgery, Vol. 2, No. 2, 1978, pp. 240-245. doi:10.1227/00006123-197805000-00010

[3] H. Suma, H. Fukumoto and A. Takeuchi, “Application of Ultrasonic Aspirator for Dissection of the Internal Mammary Artery in Coronary Artery Bypass Grafting,” Annals of Thoracic Surgery, Vol. 43, No. 6, 1987, pp. 676-677. doi:10.1016/S0003-4975(10)60251-2

[4] Y. Ito, S. Kondo, N. Sumiya, M. Yoshii, K. Otani and M. Wako, "Dermabrasion Using an Ultrasonic Surgical Aspirator," Plastic and Reconstructive Surgery, Vol. 97, No. 5, 1996, pp. 1034-1039. doi:10.1097/00006534-199604001-00024

[5] A. Rivera and S. K. Tyring, "Therapy of Cutaneous Human Papillomavirus Infections,” Dermatologic Therapy, Vol. 17, No. 6, 2004, pp. 441-448. doi:10.1111/j.1396-0296.2004.04047.x

[6] T. P. Waldinger, R. C. Wong, W. B. Taylor and J. J. Voorhees, "Cryotherapy Improves Prurigo Nodularis," Archives of Dermatology, Vol. 120, No. 12, 1984, pp. 15981600. doi:10.1001/archderm.1984.01650480060020

[7] Y. J. Park and M. S. Shin, "What Is the Best Method for Treating Osmidrosis?” Annals of Plastic Surgery, Vol. 47, No. 3, 2001, pp. 303-309. doi:10.1097/00000637-200109000-00014 